\title{
Modelo Matemático para Predição da Acidose Metabólica no Nascimento em Gestações com Diástole Zero ou Reversa
}

\author{
Mathematical Model to Predict Metabolic Acidosis at Birth in Pregnancies with \\ Absent or Reversed End-Diastolic Velocity
}

\begin{abstract}
Roseli Mieko Yamamoto Nomura, Rossana Pulcineli Vieira Francisco,
\end{abstract} Seizo Miyadahira, Marcelo Zugaib

\begin{abstract}
RESUM0
Objetivos: elaborar modelo matemático baseado em parâmetros da cardiotocografia (CTG) anteparto de repouso para predição da acidose metabólica no nascimento, caracterizada por valores do excesso de bases (BE) inferiores a-10 $\mathrm{mEq} / \mathrm{L}$, em gestações com diagnóstico de diástole zero $(D Z)$ ou reversa $(D R)$ na dopplervelocimetria das artérias umbilicais.

Métodos: foi estudada a última cardiotocografia de 127 gestantes de alto risco com diagnóstico de $D Z$ ou $D R$, pela análise visual do traçado. Os parâmetros da CTG estudados foram a variabilidade da FCF, acelerações transitórias, desacelerações e padrão pseudo-sinusoidal. Entre as caracteristicas da gravidez foram analisadas a idade gestacional, o intervalo em dias entre o diagnóstico da DZ ou DR e o parto. O modelo de regressão logística foi aplicado na determinação do melhor modelo matemático para predição da acidose.

Resultados: a acidose metabólica ocorreu em 51 casos (40,2\%). O modelo matemático para predição da acidose contemplou os seguintes parâmetros: intervalo em dias entre o diagnóstico da $D Z$ ou $D R$ e o parto $\left(\mathrm{X}_{1}\right)$, idade gestacional em semanas $\left(\mathrm{X}_{2}\right)$, variabilidade da $F C F<5 \mathrm{bpm}$ $\left(\mathrm{X}_{3}\right)$ e variabilidade da FCF entre 5 e $9 \mathrm{bpm}\left(\mathrm{X}_{4}\right)$. As variáveis de análise da variabilidade receberam valor 1 quando presente e valor $O$ quando ausente. $O$ coeficiente $z$ foi calculado pela seguinte fórmula: $z=2,2348+\left(-0,0117 \mathrm{X}_{1}\right)+\left(-0,09 \mathrm{X}_{2}\right)+\left(1,9552 \mathrm{X}_{3}\right)+\left(-0,4474 \mathrm{X}_{4}\right)$. Aplicando-se a expressão $\mathrm{p}=\mathrm{e}^{\mathrm{z}} /\left(1+\mathrm{e}^{\mathrm{z}}\right)$, a probabilidade $(\mathrm{p})$ foi calculada.

Conclusões: o modelo matemático apresentado permite calcular a probabilidade da ocorrência de acidose metabólica no nascimento, em gestações com DZ ou DR, a partir da análise visual da cardiotocografia anteparto de repouso.
\end{abstract}

PALAVRAS-CHAVE: Diástole zero ou reversa. Cardiotocografia. Excesso de bases. Acidose metabólica. Freqüência cardiaca fetal.

\section{Introdução}

O principal objetivo da avaliação da vitalidade fetal é a detecção precoce da hipoxemia fetal ${ }^{1}$, evitando-se, assim, as seqüelas neurológicas pósnatais desencadeadas pela anoxia tecidual antenatal. A insuficiência placentária constitui Setor de Vitalidade Fetal: Clínica Obstétrica do Hospital das Clinicas da Faculdade de Medicina da Universidade de São Paulo

Correspondência:

Roseli Mieko Yamamoto Nomura

Rua General Canavarro, 280 - Bairro Campestre 09070-440 - Santo André - SP

Tel: (11) 4991-2481, 4221-8778, Fax: (11) 4221-8752

e-mail: roselinomura@uol.com.br

Home Page: http://www.hcnet.usp.br/ob/ importante fator de risco para a hipoxemia, e, nessa situação, é imprescindivel assistência pré-natal especializada, oferecendo à gestante toda a propedêutica obstétrica para o diagnóstico precoce do comprometimento fetal ${ }^{2-4}$. Apesar dos avanços na área da neonatologia e da terapia intensiva neonatal, persistem, ainda, os riscos relacionados à prematuridade. O diagnóstico preciso da condição fetal permite ao perinatologista planejar o momento mais oportuno para a resolução destas gestações, confrontando sempre os riscos da prematuridade com as seqüelas neurológicas da hipoxia fetal $^{5}$.

Entre os métodos que compõem a semiologia obstétrica, tem destaque a dopplervelocimetria, 
amplamente utilizada para a avaliação da função placentária, por meio da análise dos sonogramas do cordão umbilical ${ }^{6,7}$. Este exame não invasivo permite verificar, nos casos de insuficiência placentária, o aumento da resistência ao fluxo sangüíneo na placenta, evidenciado pela redução da velocidade diastólica nas artérias umbilicais ${ }^{8}$. Nos casos graves, observa-se ausência de fluxo diastólico final (diástole zero, DZ) ou o fluxo reverso na diástole (diástole reversa, DR), os quais representam comprometimento extremo da circulação feto-placentária9 .

A cardiotocografia, um dos exames mais utilizados na avaliação do bem-estar fetal, é extremamente importante no seguimento das gestações de alto risco, principalmente nas que cursam com insuficiência placentária ${ }^{7}$. Este exame se baseia no comportamento da freqüência cardiaca fetal (FCF) e é efetuado por meio de dispositivo especial (cardiotocógrafo), o qual registra em traçados próprios a FCF, as contrações uterinas e os movimentos corpóreos fetais. As alterações no seu padrão são observadas nos diversos estados comportamentais do feto (ciclo sono-vigília), nos distúrbios de sua oxigenação ou em situações decorrentes da utilização de medicações durante a gravide ${ }^{10}$. Pela facilidade, praticidade e inocuidade inerentes do exame cardiotocográfico, este se difundiu rapidamente no mundo e, atualmente, está presente em todos os centros de referência que acompanham gestações de altorisco, tornando-se parte integrante da assistência pré-natal especializada.

As alterações nos padrões da cardiotocografia estão intimamente relacionados aos episódios de hipoxemia fetal, e a regulação dos parâmetros da FCF é dependente do adequado funcionamento do sistema nervoso autônomo ${ }^{11}$. A partir do estudo minucioso dos traçados cardiotocográficos, podese detectar quais parâmetros estão relacionados à acidose fetal. Torna-se interessante analisar a gravidade do comprometimento fetal, principalmente predizendo a possibilidade da ocorrência da acidose metabólica, caracterizada pelo consumo dos sistemas tampões, evento este relacionado ao comprometimento crônico da oxigenação fetal.

O presente estudo, realizado em gestações com insuficiência placentária grave, caracterizada pela diástole zero ou reversa à dopplervelocimetria das artérias umbilicais, tem como objetivo relacionar os parâmetros da cardiotocografia anteparto de repouso com a acidose metabólica no nascimento, caracterizada pela alteração no valor do excesso de bases (BE), e elaborar modelo matemático que seja capaz de estimar a probabilidade da ocorrência deste evento.

\section{Pacientes e Métodos}

No período compreendido entre maio de 1993 e abril de 2000, o Setor de Vitalidade Fetal da Clínica Obstétrica do Hospital da Clínicas da Faculdade de Medicina da Universidade de São Paulo (HC-FMUSP) acompanhou 249 gestações de alto risco, nas quais constatou-se DZ ou DR à dopplervelocimetria das artérias umbilicais. Todas estas pacientes foram hospitalizadas para seguimento rigoroso da vitalidade fetal, tratamento clínico e obstétrico.

Em relação aos antecedentes obstétricos, 43 pacientes $(33,9 \%)$ eram nuliparas e $84(66,1 \%)$ eram multíparas. A média da idade materna apresentou valor de 29,4 anos, com desvio padrão de 6,0 anos. Em relação às intercorrências clínicas e obstétricas associadas, constatou-se, com maior freqüência, a ocorrência de síndromes hipertensivas (101 casos, $79,5 \%$ ), seguida pela cardiopatia materna (13 casos, 10,2\%) e diabete melito (nove casos, $7,1 \%$ ).

Foram selecionados 127 casos com traçados cardiotocográficos disponiveis para revisão. Para a análise, considerou-se apenas o último traçado realizado nas 24 horas que antecederam o momento do parto, realizado no HC-FMUSP. Foram excluídos 54 casos $(21,7 \%)$ com dados incompletos de gasometria da artéria umbilical no nascimento, 33 casos de óbito fetal (13,3\%), 18 com traçado cardiotocográfico indisponível para revisão $(7,2 \%)$, nove com anomalias fetais $(3,6 \%)$ e oito gestações gemelares $(3,2 \%)$.

A dopplervelocimetria das artérias umbilicais foi realizada por via transabdominal, utilizando-se os seguintes equipamentos de ultra-sonografia: ATL Ultramark9 ou ATL HDI 3000 (Advanced Technology Laboratories Inc., Bothell, USA) e Toshiba SSA-340A (Toshiba Co., Otawara, Japan). Os exames foram realizados com a paciente em posição semi-sentada. Os sonogramas foram obtidos em períodos de inatividade fetal (ausência de movimentos corpóreos e respiratórios), utilizando-se filtros de baixos valores, entre 25 a $50 \mathrm{~Hz}$. Analisou-se o segmento do cordão umbilical próximo à placenta, aproximadamente a $5,0 \mathrm{~cm}$ de sua inserção. As duas artérias do cordão umbilical foram avaliadas e o diagnóstico de $\mathrm{DZ}$ foi confirmado quando não se observou fluxo diastólico, isto é, quando a velocidade durante a diástole tornou-se nula. A DR foi caracterizada quando observou-se fluxo negativo no canal inverso do sonograma, caracterizando o fluxo reverso. A insonação da artéria umbilical foi repetida em pelo menos cinco momentos diferentes durante o exame dopplervelocimétrico, ativando-se o dispositi- 
vo de Doppler, com mapeamento colorido de fluxo, por pelo menos um minuto.

Todos os traçados cardiotocográficos foram analisados visualmente para a elaboração deste trabalho. A cardiotocografia anteparto foi realizada com aparelhos da marca Hewlett-Packard, modelo HP 8041A (Hewlett-Packard, Boblingen, Alemanha). O exame foi realizado segundo técnica padronizada neste Setor, com a paciente em posição de semi-Fowler, velocidade de registro gráfico de um centímetro por minuto e o traçado realizado por, no mínimo, 30 minutos.

Os seguintes parâmetros cardiotocográficos foram analisados: linha de base da FCF, variabilidade da FCF, acelerações transitórias, desacelerações tardias, desacelerações variáveis, desacelerações prolongadas e padrão pseudo-sinusoidal. A classificação baseou-se nos critérios e definições estabelecidos pelo National Institute of Child Health and Human Development Research Planning Workshop ${ }^{12}$. A linha de base ou FCF basal, avaliada em batimentos por minuto, foi caracterizada pela análise visual da média aproximada da FCF no traçado, predominante num segmento de, no mínimo, 10 minutos. Foram excluidas as acelerações, desacelerações e os segmentos nos quais houvesse diferenças superiores a $25 \mathrm{bpm}$. A variabilidade da FCF foi analisada de acordo com a observação das oscilações da linha de base e foi categorizada em inferior a $5 \mathrm{bpm}$, entre 5 e $9 \mathrm{bpm}$ e superior ou igual a $10 \mathrm{bpm}$. As acelerações transitórias foram caracterizadas como ascensos da $\mathrm{FCF}$, com amplitude mínima de $15 \mathrm{bpm}$ e com duração superior a 15 segundos, independente da idade gestacional. Os traçados foram classificados de acordo com a presença ou não de, no mínimo, uma aceleração transitória. Foi caracterizada como desaceleração tardia a queda gradual da FCF, com intervalo entre início da queda e nadir superior a 30 segundos, relacionada à contração uterina e com decalagem longa, superior a 20 segundos. As desacelerações variáveis foram categorizadas em: leve (queda não atingindo $\mathrm{FCF}$ de $80 \mathrm{bpm}$ e com duração inferior a 30 segundos), moderada (queda atingindo FCF entre 70 e $80 \mathrm{bpm}$ e/ ou duração entre 30 e 60 segundos) ou grave (queda abaixo de $70 \mathrm{bpm}$ ou superior a 60 segundos). $\mathrm{Na}$ ocorrência de mais de um tipo de desaceleração variável, foi considerada somente a de maior gravidade. A desaceleração prolongada foi caracterizada quando a queda da FCF, relacionada ou não à contração uterina, apresentava-se rápida ou lenta, de amplitude variável, porém com duração superior a dois minutos. O padrão pseudo-sinusoidal foi caracterizado quando o traçado apresentava oscilações, com ondas uniformes de amplitude variando de 5 a $15 \mathrm{bpm}$, entretanto, não como o padrão sinusoidal clássico, mas com morfologia do traçado semelhante.

A gasometria da artéria umbilical foi realizada após o nascimento, em sangue colhido de segmento do cordão umbilical de cerca de $15 \mathrm{~cm}$ de comprimento, obtido por duplo clampeamento imediatamente após o nascimento. A amostra foi coletada em seringa previamente heparinizada, vedada para impedir contato com o ar ambiente e analisada a seguir. Foram verificados os parâmetros gasométricos, e para o presente estudo analisaram-se os resultados do BE. A acidose metabólica foi caracterizada quando o valor do BE foi anor$\mathrm{mal}$, isto é, quando apresentou valores inferiores $\mathrm{a}-10 \mathrm{mEq} / \mathrm{L}$.

Este estudo foi aprovado pela Comissão de Ética para Análise de Projetos de Pesquisa (CAPEPesq) da diretoria clínica do HC-FMUSP.

Inicialmente, como estratégia para construção dos modelos matemáticos, procedeu-se à análise univariada (teste do $\chi^{2}$, teste $t$ de Student e teste exato de Fisher), relacionando os parâmetros cardiotocográficos com o resultado pesquisado (alteração no BE). Desta forma, as variáveis que apresentaram associação significativa foram selecionadas para serem incluídas no modelo de regressão logística ${ }^{13}$.

A regressão logística é procedimento estatístico de análise multivariada, que possibilita o estudo de variáveis relacionadas a determinado resultado, permitindo também o controle de covariáveis durante a análise ${ }^{14}$. A construção de modelos é uma opção quando necessita-se controlar vários parâmetros simultaneamente. Este modelo estabelece estimativa de risco (probabilidade), que varia de zero a um.

Para a obtenção dos fatores preditivos foi utilizado o modelo de regressão logística com processo de seleção "stepwise". Neste procedimento estatístico, cada variável é introduzida no modelo seqüencialmente, de acordo com a maior correlação positiva observada, sendo então testadas quanto à sua permanência ou não no modelo matemático. Variáveis independentes potencialmente classificáveis como 'variáveis de confusão' foram incluídas na regressão. Os parâmetros do modelo foram estimados utilizando-se o método de probabilidade máxima, e os coeficientes que ofereceram melhor predição foram selecionados ${ }^{15}$. Para mais de uma variável independente, o modelo é elaborado a partir da fórmula: $p=e^{z} /\left(1+e^{z}\right)$, onde $z$ é a combinação linear: $z=B_{o}+B_{1} X_{1}+B_{2} X 2 \ldots . B_{p} X_{p}$; o risco individual pode ser estimado ( $p=$ probabilidade). Todas as análises foram realizadas com o programa SAS 6.11. O nivel de significância utilizado para os testes foi de $5 \%$. 


\section{Resultados}

Entre os 127 casos analisados no presente estudo, $76(59,8 \%)$ apresentaram valores normais de $\mathrm{BE}(\mathrm{BE} \geq-10 \mathrm{mEq} / \mathrm{L})$ na gasometria de artéria umbilical no nascimento. A acidose metabólica caracterizada por valores anormais no $\mathrm{BE}$ $(\mathrm{BE}<-10 \mathrm{mEq} / \mathrm{L})$ foi constatada em 51 casos $(40,2 \%)$. Todos os partos foram cesáreos, e a idade gestacional no nascimento variou de 26 semanas e 6 dias a 36 semanas e 4 dias. A média da idade gestacional no nascimento no grupo com $\mathrm{BE}$ normal foi de 31,8 semanas ( $\mathrm{DP}=2,4 \mathrm{sem})$. Nos casos com $\mathrm{BE}$ alterado, a média da idade gestacional no nascimento foi de 31,1 semana ( $\mathrm{DP}=2,3 \mathrm{sem}$ ). Quanto ao intervalo (em dias) entre o diagnóstico de DZ ou DR e o parto, não foram observadas diferenças significativas entre os grupos que apresentaram ou não alterações no BE, sendo respectivamente de 5,8 e 8,4 dias, com respectivos desvios-padrão de 11,9 e 14,2 dias.

A ocorrência de DR não apresentou associa- ção com o tipo de resultado do BE no nascimento. Observou-se que a proporção de casos com DR nos casos com valores normais de $\mathrm{BE}$ foi de $23,7 \%$ (18 casos), e nos casos com acidose metabólica foi de $37,3 \%$ (19 casos).

A análise univariada da associação entre os parâmetros cardiotocográficos e as alterações nos valores do BE está na Tabela 1 . Em relação à classificação dos casos de acordo com a FCF basal, não foi constatada relação entre os grupos classificados de acordo com a linha de base e a ocorrência de alterações no BE. Na análise da variabilidade, podemos verificar associação significativa entre a redução da variabilidade e a ocorrência de valores alterados no $B E$ no nascimento $(p=0,001)$. Podemos observar ainda associação estatisticamente significativa $(p=0,001)$ entre a ocorrência de desacelerações tardias e anormalidades no BE. Porém, a presença de desacelerações variáveis, desacelerações prolongadas e do padrão pseudosinusoidal não apresentou correlação significativa com a ocorrência de acidose metabólica.

Tabela 1 - Distribuição dos parâmetros cardiotocográficos da FCF de acordo com os resultados do excesso de bases (BE) no nascimento, em gestações com diástole zero ou reversa na dopplervelocimetria das artérias umbilicais.

\begin{tabular}{|c|c|c|c|c|c|}
\hline \multirow{2}{*}{$\begin{array}{l}\text { Parâmetro cardiotocográfico } \\
\text { FCF basal, média (DP) em bpm }\end{array}$} & \multicolumn{2}{|c|}{$\begin{array}{c}\text { BE Normal } \\
B E \geq-10(n=76)\end{array}$} & \multicolumn{2}{|c|}{$\begin{array}{c}\text { BE Anormal } \\
\text { BE }<-10(n=51)\end{array}$} & \multirow{2}{*}{$\begin{array}{c}p \\
\text { NS }\end{array}$} \\
\hline & 139,6 & $(8,8)$ & 140,0 & $(8,7)$ & \\
\hline \multicolumn{6}{|l|}{ FCF basal, n (\%) } \\
\hline$<120$ bpm & 1 & $(50,0)$ & 1 & $(50,0)$ & \multirow{3}{*}{ NS } \\
\hline $120-155$ bpm & 74 & $(60,2)$ & 49 & $(39,8)$ & \\
\hline$>155$ bpm & 1 & $(50,0)$ & 1 & $(50,0)$ & \\
\hline \multicolumn{6}{|l|}{ AT, n (\%) } \\
\hline Presente & 21 & $(84,0)$ & 4 & $(16,0)$ & \multirow[b]{2}{*}{$<0,01$} \\
\hline Ausente & 55 & $(53,9)$ & 47 & $(46,1)$ & \\
\hline \multicolumn{6}{|l|}{ Variabilidade, $n(\%)$} \\
\hline$<5$ bpm & 5 & $(19,2)$ & 21 & $(80,8)$ & \multirow{3}{*}{0,001} \\
\hline $5-9$ bpm & 26 & $(63,4)$ & 15 & $(36,6)$ & \\
\hline$\geq 10 \mathrm{bpm}$ & 45 & $(75,0)$ & 15 & $(25,0)$ & \\
\hline \multicolumn{6}{|l|}{ Desaceleração tardia, n (\%) } \\
\hline Presente & 24 & $(43,6)$ & 31 & $(56,4)$ & \multirow[b]{2}{*}{0,001} \\
\hline ausente & 52 & $(72,2)$ & 20 & $(27,8)$ & \\
\hline \multicolumn{6}{|l|}{ Desaceleração variável, n (\%) } \\
\hline Grave & 13 & $(44,8)$ & 16 & $(55,2)$ & \multirow{4}{*}{ NS } \\
\hline Moderada & 9 & $(56,3)$ & 7 & $(43,7)$ & \\
\hline Leve & 24 & $(63,2)$ & 14 & $(36,8)$ & \\
\hline Ausente & 30 & $(68,2)$ & 14 & $(31,8)$ & \\
\hline \multicolumn{6}{|l|}{ Desaceleração prolongada, n (\%) } \\
\hline Presente & 11 & $(64,7)$ & 6 & $(35,3)$ & \multirow[b]{2}{*}{ NS } \\
\hline Ausente & 65 & $(59,1)$ & 45 & $(40,9)$ & \\
\hline \multicolumn{6}{|l|}{ Padrão pseudo-sinusoidal, $\mathrm{n}(\%)$} \\
\hline Presente & 2 & $(40,0)$ & 3 & $(60,0)$ & \multirow[b]{2}{*}{ NS } \\
\hline ausente & 74 & $(60,7)$ & 48 & $(39,3)$ & \\
\hline
\end{tabular}


Para o estudo multivariado dos parâmetros cardiotocográficos, foram selecionadas as variáveis que se apresentaram relacionadas a valores alterados do BE no nascimento, para serem introduzidas no modelo de regressão logística. Deste modo, os seguintes parâmetros foram selecionados: niveis da variabilidade da FCF (inferior a $5 \mathrm{bpm}$, entre 5 e $9 \mathrm{bpm}$, igual ou superior a 10 $\mathrm{bpm})$, presença de acelerações transitórias e a ocorrência de desacelerações tardias. Foram adicionadas também as variáveis idade gestacional no nascimento e o intervalo entre o diagnóstico da DZ ou DR e o parto.

Pelo procedimento 'stepwise', os dados foram analisados para a estimativa da probabilidade máxima de alteração no BE no nascimento. Para elaborar o modelo final da análise dos parâmetros cardiotocográficos na predição de alteração no $\mathrm{BE}$ em casos de DZ ou DR, foram contempladas, pela regressão logística, como de melhor valor preditivo, as variáveis relacionadas com a variabilidade da FCF. Foram mantidas no modelo final duas variáveis de confusão consideradas importantes por se relacionarem com a variabilidade da FCF: o intervalo entre o diagnóstico da DZ ou DR e o parto $\left(X_{1}\right)$, e a idade gestacional no nascimento $\left(X_{2}\right)$.

Na Tabela 2 estão mostrados os valores dos parâmetros estimados para cada variável mantida no modelo matemático, com respectivos valores de probabilidades (p) e razão de chances (odds ratio). Destaca-se o valor de 7,065 do odds ratio para variabilidade da FCF inferior a $5 \mathrm{bpm}$.

Tabela 2 - Graus de liberdade, parâmetro estimado, erro padrão, probabilidade e odds ratio e intervalo de confiança a 95\% das variáveis selecionadas na regressão logística, para a predição da acidose metabólica no nascimento em gestações com diástole zero (DZ) ou reversa na dopplervelocimetria das artérias umbilicais.

\begin{tabular}{lcccccc}
\hline Variável & g.l. & Parâmetro estimado & Erro padrão & $\mathbf{p}$ & Odds ratio & Intervalo de confiança 95\% \\
\hline Intercepto & 1 & 2,3248 & 2,8320 & 0,4117 & - & \\
Intervalo entre o diagnóstico & 1 & $-0,0117$ & 0,0169 & 0,4865 & 0,988 & 0,$956 ; 1,022$ \\
da DZ e o parto & & & & & & \\
Idade gestacional no parto & 1 & $-0,0900$ & 0,0898 & 0,3161 & 0,914 & 0,$767 ; 1,090$ \\
Variabilidade inferior a 5 bpm & 1 & 1,9552 & 0,5989 & 0,0011 & 7,065 & 2,$184 ; 22,853$ \\
Variabilidade de 5 a 9 bpm & 1 & $-0,4474$ & 0,4512 & 0,3214 & 0,639 & 0,$264 ; 1,548$ \\
\hline
\end{tabular}

g.l. = graus de liberdade

O modelo matemático final para estimar a probabilidade (p) da ocorrência de acidose no nascimento incluiu as variáveis de confusão e as seguintes: variabilidade inferior a $5 \mathrm{bpm}\left(X_{3}\right)$ e variabilidade da FCF 5-9 bpm $\left(X_{4}\right)$. Cada variável $\left(X_{3} \mathrm{e}\right.$ $X_{4}$ ) assume valor 1 quando presente ou 0 quando ausente. O valor de $z$ pode ser calculado pela fórmula abaixo:

$z=2,2348+\left(-0,0117 \cdot X_{1}\right)+\left(-0,09 \cdot X_{2}\right)+\left(1,9552 \cdot X_{3}\right)$

$+\left(-0,4474 . X_{4}\right)$

Aplicando a expressão $p=e^{z} /\left(1+e^{z}\right)$, é possível estimar a probabilidade $(\mathrm{p})$ da ocorrência de alterações no equilíbrio ácido-básico fetal.

Na Tabela 3 estão listados os valores da acurácia, sensibilidade e especificidade do modelo, de acordo com o valor de probabilidade obtida. Destaca-se, nesta tabela, a acurácia de 72,4\% do modelo quando se obtêm valores de probabilidade entre 0,48 e 0,72, com elevada especificidade de $93 \%$.

\section{Discussão}

A insuficiência placentária grave, diagnosticada pela ocorrência de DZ ou DR, tem sido freqüentemente associada a resultados perinatais adversos, com elevada incidência de hipoxemia e acidemia fetais. A mortalidade perinatal é elevada, variando de 15 a $62 \%$ nas maiores casuísticas referidas na literatura ${ }^{16,17}$. Na Clínica Obstétrica do HC-FMUSP, no período compreendido entre 1992 e 1999, a mortalidade perinatal observada entre estes casos foi de $35,8 \%{ }^{18}$. Devido à gravidade que representa este diagnóstico, os casos exigem acompanhamento obstétrico diferenciado e intensivo, principalmente porque a falência placentária traz graves repercussões ao produto conceptual, freqüentemente em períodos críticos da gestação, no limiar da viabilidade, e relacionase a doenças maternas graves.

Clinicamente, o limite a partir do qual a acidose fetal é considerada significativa não é bem definido, de tal modo que vários estudos têm sugerido diferentes classificações para o diagnóstico da acidose fetal patológica. Aceita-se que existam várias e complexas interações no metabolis- 
mo fetal que regulem o $\mathrm{pH}$ e o consumo das bases tampões em face do sofrimento fetal ${ }^{19}$. A evolução do padrão cardiotocográfico do feto normalmente oxigenado para o observado na hipoxemia, principalmente nas hipoxemias com magnitude suficiente para produzir o dano neurológico, não é bem compreendida ${ }^{20,21}$. Do mesmo modo, caracteristicas individuais do organismo fetal devem influenciar os mecanismos de lesão celular decorrente da hipoxia e acidose tecidual.

Tabela 3 - Acurácia, sensibilidade e especificidade de acordo com o valor de probabilidade obtido pelo modelo matemático de predição de acidose metabólica no nascimento em gestações com diástole zero ou reversa na dopplervelocimetria das artérias umbilicais.

\begin{tabular}{cccc}
\hline Probabilidade & Acurácia & Sensibilidade & Especificidade \\
\hline 0.040 & 40.2 & 100.0 & 0.0 \\
0.060 & 39.4 & 98.0 & 0.0 \\
0.080 & 39.4 & 98.0 & 0.0 \\
0.100 & 39.4 & 98.0 & 0.0 \\
0.120 & 39.4 & 98.0 & 0.0 \\
0.140 & 39.4 & 98.0 & 0.0 \\
0.160 & 39.4 & 98.0 & 0.0 \\
0.180 & 39.4 & 98.0 & 0.0 \\
0.200 & 39.4 & 92.2 & 3.9 \\
0.220 & 42.5 & 90.2 & 10.5 \\
0.240 & 48.8 & 86.3 & 23.7 \\
0.260 & 53.5 & 80.4 & 35.5 \\
0.280 & 57.5 & 76.5 & 44.7 \\
0.300 & 55.9 & 66.7 & 48.7 \\
0.320 & 59.1 & 62.7 & 56.6 \\
0.340 & 60.6 & 56.9 & 63.2 \\
0.360 & 65.4 & 52.9 & 73.7 \\
0.380 & 65.4 & 47.1 & 77.6 \\
0.400 & 66.1 & 43.1 & 81.6 \\
0.420 & 66.9 & 41.2 & 84.2 \\
0.440 & 67.7 & 41.2 & 85.5 \\
0.460 & 71.7 & 41.2 & 92.1 \\
0.480 & 72.4 & 41.2 & 93.4 \\
0.500 & 72.4 & 41.2 & 93.4 \\
0.520 & 72.4 & 41.2 & 93.4 \\
0.540 & 72.4 & 41.2 & 93.4 \\
0.560 & 72.4 & 41.2 & 93.4 \\
0.580 & 72.4 & 41.2 & 93.4 \\
0.600 & 72.4 & 41.2 & 93.4 \\
0.620 & 72.4 & 41.2 & 93.4 \\
0.640 & 72.4 & 41.2 & 93.4 \\
0.660 & 72.4 & 41.2 & 93.4 \\
0.680 & 72.4 & 41.2 & 93.4 \\
0.700 & 72.4 & 41.2 & 93.4 \\
0.720 & 72.4 & 41.2 & 93.4 \\
0.740 & 71.7 & 39.2 & 93.4 \\
0.760 & 70.1 & 35.3 & 93.4 \\
0.780 & 68.5 & 29.4 & 94.7 \\
0.800 & 67.7 & 27.5 & 94.7 \\
0.820 & 63.8 & 17.6 & 94.7 \\
0.840 & 59.1 & 3.9 & 96.1 \\
0.860 & 59.1 & 0.0 & 98.7 \\
0.880 & 59.8 & 0.0 & 100.0 \\
\hline & & & \\
\hline
\end{tabular}

A análise dos parâmetros gasométricos permite avaliar o estado metabólico e as alterações relacionadas ao desequilíbrio ácido-básico fetal. O excesso de bases negativo corresponde ao consumo das bases dos sistemas tampões fetais, principalmente do bicarbonato, e valores do BE inferiores a $-10 \mathrm{mEq} / \mathrm{L}$ correspondem aos casos de maior gravidade, refletindo geralmente a instalação da acidose metabólica fetal. Observa-se também que a hipoxemia e a hipercapnia acompanham geralmente os casos de acidose fetal, porém, freqüentemente, estes parâmetros sofrem alterações rápidas em seus valores, decorrentes principalmente dos procedimentos relacionados ao momento do parto, limitando a utilização dos seus valores na avaliação da condição fetal ${ }^{19}$. Os valores do $\mathrm{BE}$ não sofrem influências de eventos agudos, favorecendo a sua utilização para melhor avaliação da acidose metabólica fetal.

Na hipoxemia crônica, os fetos evoluem com adaptações do sistema nervoso autônomo a longo prazo, interferindo provavelmente na regulação da FCF basal. Há longa data sabe-se que os parâmetros cardiotocográficos podem espelhar o adequado funcionamento do sistema nervoso autônomo, e conseqüentemente do sistema nervoso central ${ }^{22}$. Porém, o estudo simultâneo de múltiplas variáveis dificulta a interpretação lógica dos resultados observados, incutindo um caráter subjetivo na análise dos traçados. Deste modo, tentando padronizar e facilitar a interpretação dos traçados, com rigor científico pelo uso de procedimentos estatísticos adequados, optou-se pela análise com o modelo de regressão logística. Este procedimento estatístico permite analisar os dados com o máximo de cuidado e elaborar estimativas de risco, em forma de probabilidade ${ }^{15}$. O modelo matemático foi portanto construído para predizer a ocorrência de acidose metabólica no nascimento, caracterizada pela ocorrência de valores alterados do excesso de bases, baseando-se na análise de cada parâmetro cardiotocográfico previamente categorizado.

As variáveis chamadas 'de confusão' foram contempladas nestes modelos, a saber: idade gestacional no nascimento e o intervalo entre o diagnóstico de $\mathrm{DZ}$ e o parto. Sabe-se que a regulação da FCF é realizada pelo sistema nervoso autônomo, que por sua vez pode não estar completamente amadurecido em gestações prematuras. Isto pode alterar alguns parâmetros analisados na cardiotocografia anteparto de repouso. A introdução desta variável nos modelos de regressão procurou contemplar alterações que são decorrentes da própria prematuridade, sem influenciar nos resultados obtidos quando as alterações cardiotocográficas são decorrentes da hipoxia e acidemia fetais. Do mesmo modo, a variável que avalia o intervalo 
entre o diagnóstico da DZ ou DR e o parto foi contemplada, pois este período reflete o tempo em que o feto está submetido a um regime de insuficiência placentária grave, piorando o seu prognóstico no período neonatal.

O método de análise da regressão logística permite a construção dos modelos matemáticos ${ }^{13}$, que podem ser introduzidos em sistemas computadorizados ou planilhas eletrônicas, possibilitando o cálculo da probabilidade da ocorrência de um evento, com rapidez e facilidade. Para a construção dos modelos, utilizou-se do programa SAS 6.11, e as variáveis que apresentaram correlação estatística com o diagnóstico pesquisado foram introduzidas no programa. A análise final aponta os parâmetros que apresentam melhor desempenho na predição do resultado pesquisado. Pode-se observar elevado risco associado à redução da variabilidade da FCF, com razão de chances (odds ratio) superior a 7 para predição da acidose metabólica quando a variabilidade for inferior a 5 $\mathrm{bpm}$. Isto significa um risco 7 vezes maior para acidose metabólica nesta situação, se comparado aos casos com variabilidade superior ou igual a 5 bpm, em gestações com DZ ou DR.

Este modelo tem aplicação prática imediata, uma vez que, ao introduzir os valores da análise visual do traçado cardiotocográfico, programas computadorizados calculam automaticamente o risco da ocorrência de acidemia no nascimento. É possivel também determinar, para cada valor de probabilidade estimada, a acurácia, a sensibilidade e a especificidade do teste aplicado, de forma que podem-se estudar os valores limites de acordo com a necessidade de níveis de probabilidade com elevada sensibilidade ou especificidade, para que o modelo apresente seu melhor desempenho. Este aspecto do programa torna-se interessante quando analisamos casos com prematuridade extrema e elevada morbidade neonatal, em que a prorrogação da gestação poderá propiciar melhores condições de maturidade fetal. Gestações que cursam com DZ ou DR precocemente, nos limites da viabilidade fetal, se beneficiarão de níveis de probabilidade com elevada especificidade, uma vez que os riscos da prematuridade extrema devem ser considerados ${ }^{23}$. Em gestações mais tardias, no terceiro trimestre, torna-se interessante a utilização de níveis de probabilidade com maior sensibilidade, uma vez que a mortalidade e o risco de seqüelas da prematuridade se reduzem.

A limitação deste modelo desenvolvido consiste na obrigatoriedade de se aplicar esta fórmula somente aos casos de DZ ou DR, em gestações compreendidas entre 26 a 37 semanas, a partir da análise visual dos traçados cardiotocográficos e após treinamento adequado que permita classi- ficar os parâmetros analisados. A utilização em condições diferentes pode determinar erros diagnósticos que poderão comprometer o prognóstico neonatal.

É interessante notar que, no modelo final, não foi contemplada a presença ou não de acelerações transitórias na determinação do risco para acidose, priorizando a análise da variabilidade. Provavelmente, os casos com variabilidade normal da FCF estão englobando os que apresentam as acelerações transitórias.

Poucos estudos utilizam modelos matemáticos para análise dos parâmetros cardiotocográficos, e nenhum em gestações com DZ ou DR. A Clinica Obstétrica do HC-FMUSP constitui serviço de referência para atendimento de gestantes de alto risco e recebe, anualmente, cerca de 35 casos de DZ ou DR. Nos últimos nove anos, o Setor de Vitalidade Fetal aprimorou o seu sistema de coleta e armazenamento de dados, possibilitando a realização de pesquisas nesta área, aplicandose protocolos sob supervisão da mesma equipe. Para utilização do modelo de regressão logística, é necessário um número grande de casos com casuística completa, o que explica a dificuldade de se realizar trabalhos semelhantes ao presente estudo.

Novas perspectivas surgem com estes métodos de predição de acidose, principalmente a possibilidade de testá-los prospectivamente e confrontar a eficácia destes modelos com outros já desenvolvidos neste Setor. De particular interesse, são também os estudos do perfil hemodinâmico fetal ${ }^{24}$, que apresentam correlação com a acidose no nascimento, podendo predizer os riscos para as diferentes faixas de anormalidades no $\mathrm{pH}$ da artéria umbilical ${ }^{25}$. A associação destes testes parece ser muito promissora, principalmente porque estas gestações, de elevada gravidade, necessitam de embasamento fisiopatológico para o correto diagnóstico da condição de oxigenação fetal e para análise da resposta metabólica do produto conceptual.

Tendo em vista a extrema complexidade e a estreita faixa de segurança no acompanhamento dos casos de DZ ou DR, as dificuldades se configuram diante de duas situações preocupantes: a carência no fornecimento de oxigênio ao feto e a prematuridade trazendo potenciais riscos para o período neonatal. Rico arsenal propedêutico, quando disponivel e racionalmente utilizado, possibilita o diagnóstico precoce da hipoxemia fetal, um passo importante para se evitarem ou minimizarem os danos neurológicos no recém-nascido.

Concluindo, vale reiterar que, a partir do modelo final da regressão logística, foi possivel elaborar modelo matemático que permite o cálculo 
da probabilidade de alterações no excesso de bases no nascimento, aplicando-se a fórmula de probabilidade em gestantes com o diagnóstico de DZ ou DR nas artérias umbilicais. Conhecendo-se as características do traçado e utilizando-se de dados da interpretação visual da cardiotocografia, podemos obter a probabilidade da ocorrência da acidose metabólica no nascimento.

\section{ABSTRACT}

Purpose: to develop a mathematical model based on the fetal heart rate (FHR) monitoring to predict metabolic acidosis at birth (base excess $<-10 \mathrm{mEq} / \mathrm{L}$ ), in pregnancies with absent or reversed end-diastolic velocity (AREDV) in the umbilical arteries.

Methods: the last FHR tracing of 127 AREDV cases was studied by visual analysis. The analyzed parameters included: gestational age, interval between AREDV diagnosis and delivery, FHR variability, FHR accelerations, decelerations, and sinusoidal- like pattern. Multivariate logistic regression was applied to find the best mathematical model to predict acidosis.

Results: metabolic acidosis at birth occurred in 51 cases (40.2\%). The model included the parameters: interval between AREDV diagnosis and delivery $\left(X_{1}\right)$, gestational age in weeks $\left(X_{2}\right), F H R$ variability $<5 \mathrm{bpm}\left(X_{3}\right)$, and FHR variability 5-9 bpm $\left(X_{4}\right)$. To each variability parameter was assigned the value of 1 when present or 0 when absent. The $z$ value is: $\mathrm{z}=2.2348+\left(-0.0117 X_{1}\right)+\left(-0.09 X_{2}\right)+\left(1.9552 X_{3}\right)+$ $\left(-0.4474 X_{4}\right)$. By applying the expression $p=e^{z} /\left(1+e^{z}\right)$, the probability is estimated.

Conclusion: the mathematical model allowed us to estimate the probability of metabolic acidosis at birth, in pregnancies with AREDV, studying FHR-monitoring parameters.

KEY WORDS: Absent or reversed end-diastolic velocity. Cardiotocography. Metabolic acidosis. Fetal heart rate.

\section{Referências}

1. ACOG Practice Bulletin. Antepartum fetal surveillance. Number 9, October 1999. Clinical management guidelines for obstetriciangynecologists. Int J Gynaecol Obstet 2000; 68:175-85.

2. Visser GH, Sadovsky G, Nicolaides KH. Antepartum heart rate patterns in small-for-gestational-age third-trimester fetuses: Correlations with blood gas values obtained at cordocentesis. Am J Obstet Gynecol 1990;162:698-703.
3. Okamura M, Itakura A, Kurauchi O, Hayakawa F, Mizutani S, Tomoda Y. Fetal heart rate patterns associated with periventricular leukomalacia. Int $\mathrm{J}$ Gynaecol Obstet 1997; 56:13-8.

4. Westgate JA, Gunn AJ, Gunn TR. Antecedents of neonatal encephalopathy with fetal acidaemia at term. Br J Obstet Gynaecol 1999;106:774-82.

5. Black RS, Campbell S. Cardiotocography versus Doppler. Ultrasound Obstet Gynecol 1997; 9:148-51.

6. Baschat AA, Weiner CP. Umbilical artery Doppler screening for detection of the small fetus in need of antepartum surveillance. Am J Obstet Gynecol 2000; 182:154-8.

7. Haley J, Tuffnell DJ, Johnson N. Randomised controlled trial of cardiotocography versus umbilical artery Doppler in the management of small for gestational age fetuses. Br J Obstet Gynaecol 1997; 104:431-5.

8. Trudinger BJ, Giles WB, Cook CM, Bombardieri J, Collins L. Fetal umbilical artery flow velocity waveforms and placental resistance: clinical significance. Br J Obstet Gynaecol 1985; 92:23-30.

9. Salafia CM, Pezzulo JC, Minior VK, Divon MY. Placental pathology of absent and reversed enddiastolic flow in growth-restricted fetuses. Obstet Gynecol 1997; 90:830-6.

10.Zugaib M, Behle I. Fisiologia cardiovascular aplicada à monitoração fetal. In: Zugaib M, Behle I, editores. Monitoração Fetal Eletrônica. $1^{\text {a }}$ ed. São Paulo: Roca; 1981. p.25-53.

11.Zugaib M, Forsythe AB, Nuwayhid B, et al. Mechanisms of beat-to-beat variability in the heart rate of the neonate lamb. II. Effects of hypoxia. Am J Obstet Gynecol 1980; 138:453-8.

12.National Institute of Child Health and Human Development Research Planning Workshop. Electronic fetal heart rate monitoring: research guidelines for interpretation. Am J Obstet Gynecol 1997; 177:1385-90.

13. Hosmer DW, Lemeshow S. Applied logistic regression. New York: John Wiley \& Sons; 1989.

14.Vandewalle J. Advanced statistical techniques: how to use your data more effectively? Ultrasound Obstet Gynecol 1999; 13:8-10.

15.Peterson HB, Kleinbaum DG. Interpreting the literature in obstetrics and gynecology: II. Logistic regression and related issues. Obstet Gynecol 1991; 78:717-20.

16.Karsdorp VH, van Vugt JM, van Geijn HP, et al. Clinical significance of absent or reversed end diastolic velocity waveforms in umbilical artery. Lancet 1994; 344:1664-8.

17.Kurkinen-Räty M, Kivelä A, Jouppila P. The clinical significance of an absent end-diastolic velocity in the umbilical artery detected before the 34th week of pregnancy. Acta Obstet Gynecol Scand 1997; 76:398-404. 
18. Yamamoto RM, Francisco RPV, Miyadahira S, Chuba CC, Zugaib M. Fatores prognósticos para o óbito perinatal em gestações com diástole zero ou reversa na dopplervelocimetria das artérias umbilicais. Rev Bras Ginecol Obstet 2000; 22:353-63.

19.Kjellmer I. Fetal biochemical reactions to hypoxia. Eur J Obstet Gynecol Reprod Biol 1991; 42 Suppl:S21-3.

20.Hanson MA. Do we now understand the control of the fetal circulation? Eur J Obstet Gynecol Reprod Biol 1997; 75:55-61.

21.Ikeda T, Murata Y, Quilligan EJ, et al. Fetal heart rate patterns in postasphyxiated fetal lambs with brain damage. Am J Obstet Gynecol 1998; 179:1329-37.
22. Fouron LC. Does the brain-sparing effect compensate for hypoxemia? In: Arbeille PH, Maulik D, Laurini RN, editors. Fetal Hypoxia. 1st ed. New York: Parthenon; 1999. p.89-98.

23.Zelop CM, Richardson DK, Heffner LJ. Outcomes of severely abnormal umbilical artery Doppler velocimetry in structurally normal singleton fetuses. Obstet Gynecol 1996; 87:434-8.

24.Hecher K, Hackelöer BJ. Cardiotocogram compared to Doppler investigation of the fetal circulation in the premature growth-retarded fetus: longitudinal observations. Ultrasound Obstet Gynecol 1997; 9:152-61.

25.Francisco RPV. Predição da acidose no nascimento em gestações com diástole zero ou reversa à dopplervelocimetria das artérias umbilicais [dissertação]. São Paulo: Universidade de São Paulo; 1998.

\title{
IX Congresso de Ginecologia e Obstetrícia da Região Sudeste da FEBRASGO XXVI Congresso de Ginecologia e Obstetrícia da SGORJ
}

\author{
24 a 27 de julho de 2002
}

Rio de Janeiro-RJ

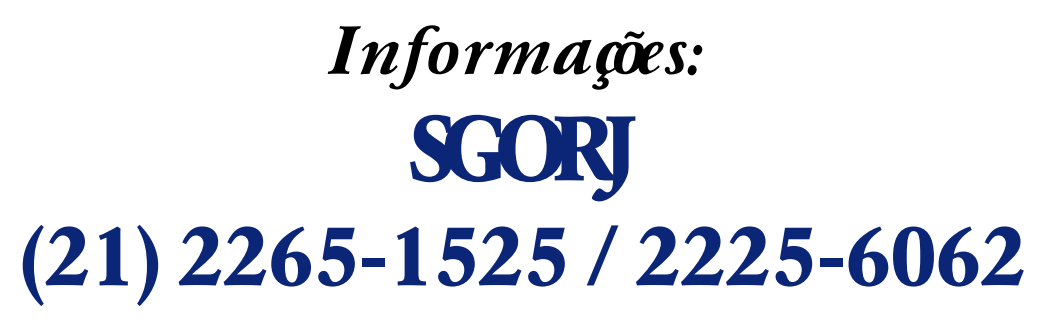

